\section{Tear film lipid layer thickness and ocular comfort after meibomian therapy via latent heat with a novel device in normal subjects}

M Mitra1', GJ Menon'1, A Casini², S Hamada1, D Adams², C Ricketts ${ }^{3}$, ET Fuller ${ }^{4}$ and JR Fuller ${ }^{1}$

\begin{abstract}
Aim This study measures changes in tear film lipid layer thickness (LLT) and ocular comfort in normal subjects after $10 \mathrm{~min}$ use of a novel device, which delivers meibomian therapy with latent heat. The device is designed to promote the release of meibomian sebum into the tear film by delivering latent heat to the eyelids, thus thickening the lipid layer. Normal lid movements are maintained, facilitating resurfacing of the tear film.
\end{abstract}

Method A prospective, controlled, observer masked, single intervention trial in which 24 normal subjects were randomised into three groups. Group I underwent $\mathbf{1 0}$ min treatment with the activated device, Group II used the inactivated device for the same duration of time, and Group III had no intervention. The LLT of each subject was measured with a Keeler Tearscope ${ }^{\mathbb{R}}$ prior and subsequent to the 10 -min period. Subjective alteration in ocular comfort was also assessed.

Results Seven of eight subjects $(87.5 \%)$ in Group I exhibited an increase in LLT. The mean LLT in this group showed a statistically significant increase (left eyes 1.0 levels, $P<0.001$, right eyes 0.9 levels, $P<0.003$ ) compared to Groups II and III. Six of eight subjects $(75 \%)$ using the activated device experienced subjective improvement in ocular comfort.

Conclusion Meibomian therapy with this device increases LLT in normal individuals. This implies a more stable tear film, reflected in subjective improvement in ocular comfort.

Eye (2005) 19, 657-660. doi:10.1038/sj.eye.6701611

Published online 27 August 2004
Keywords: meibomian gland therapy; lipid layer thickness; ocular comfort

\section{Introduction}

The lipid layer of the tear film, derived principally from the meibomian glands, plays a vital role in the maintenance of tear film stability. ${ }^{1}$ Increased thickness of this lipid layer has been shown to correlate with improved stability of the tear film. ${ }^{2}$ Different methods for increasing lipid layer thickness (LLT) have been shown to be effective including manual expression, massage, and the application of heat. ${ }^{3-5}$ Current therapy for patients with obstructive meibomian gland dysfunction and evaporative dry eye includes meibomian therapy with heat, often delivered with a hot flannel placed over the eyelids. However, many patients find this inconvenient - and poor patient compliance is an obstacle to effective therapy.

We have designed a novel device to deliver meibomian therapy with heat. This study evaluates the effect of this device on LLT and ocular comfort in normal subjects.

\section{Materials and methods}

With formal approval from the South and West Devon Research Ethics Committee, a randomised, controlled, prospective, observer masked, single intervention trial was conducted, involving 24 normal subjects, none of whom were contact lens wearers. All subjects had their LLT measured with the Keeler Tearscope $^{\circledR}$ (Keeler Instruments, Clewer Hill
${ }^{1}$ Department of Ophthalmology Royal Eye Infirmary Plymouth, UK

${ }^{2}$ Department of Optometry Royal Eye Infirmary

Plymouth, UK

${ }^{3}$ School of Mathematics and Statistics

University of Plymouth, UK

${ }^{4}$ Design Engineer

Christchurch

New Zealand

Correspondence: JR Fuller Department of Ophthalmology Royal Eye Infirmary Apsley Road Plymouth PL4 6PL, UK Tel: + 441752315127 Fax: + 441752254162 E-mail: robfulleruk@hotmail.com

Received: 9 January 2004 Accepted: 13 April 2004 Published online: 27 August 2004 
Road, Windsor, Berkshire SL4 4AA, UK) prior to and $10 \mathrm{~min}$ after intervention. This instrument utilises a diffuse cold light source, which in conjunction with a nonilluminated biomicroscope affords observation of the superficial lipid layer of the tear film by interference reflection. Five grades of thicknesses of the normal lipid layer are recognised: (i) open meshwork, (ii) closed meshwork, (iii) flow, (iv) amorphous, and (v) colour fringes. ${ }^{6}$

The subjects, aged between 23 and 62 years (mean 41 years), were randomised into three groups of eight. Group I (two male, six female, mean age 41 years) underwent $10 \mathrm{~min}$ treatment with the activated device set at $50^{\circ} \mathrm{C}$. Group II (three male, five female, mean age 36 years) used the inactivated device for the same duration of time, and Group III (two male, six female, mean age 47 years) underwent no intervention.

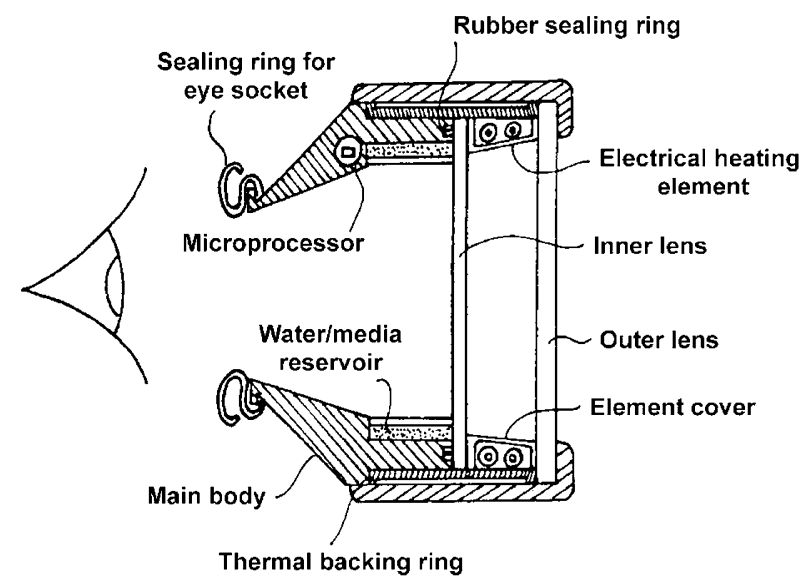

Figure 1 Design concept.
An experienced investigator (AC), masked to randomisation and intervention, recorded pre- and posttrial LLT. The subjects also had their ocular comfort assessed with respect to whether their eyes felt (a) much less comfortable, (b) moderately less comfortable, (c) minimally less comfortable, (d) unchanged, (e) minimally more comfortable, (f) moderately more comfortable, or (g) much more comfortable, after the trial.

\section{The device}

This device (Figures 1 and 2) consists of a pair of specially designed goggles, fitted against the upper part of the face so as to cover the eyes and eyelids. It includes a liquid reservoir and means for heating and humidifying the air around the eyes. A mechanism to prevent condensation is also incorporated, in order to enable clear vision for the continuance of sedentary activity. The humidity optimises efficient heat transfer to the skin of the lids by conduction and convection. More importantly, a thermal siphon is established, in which water evaporated from the heated surface of the device, condenses on the relatively cool eyelids with transfer of latent heat. Heat is therefore transferred at a lower temperature, thus avoiding the discomfort associated with radiant heat. Excess condensed water is recycled in the device.

\section{Results}

LLT was ranked according to the five recognised normal grades (Table 1).

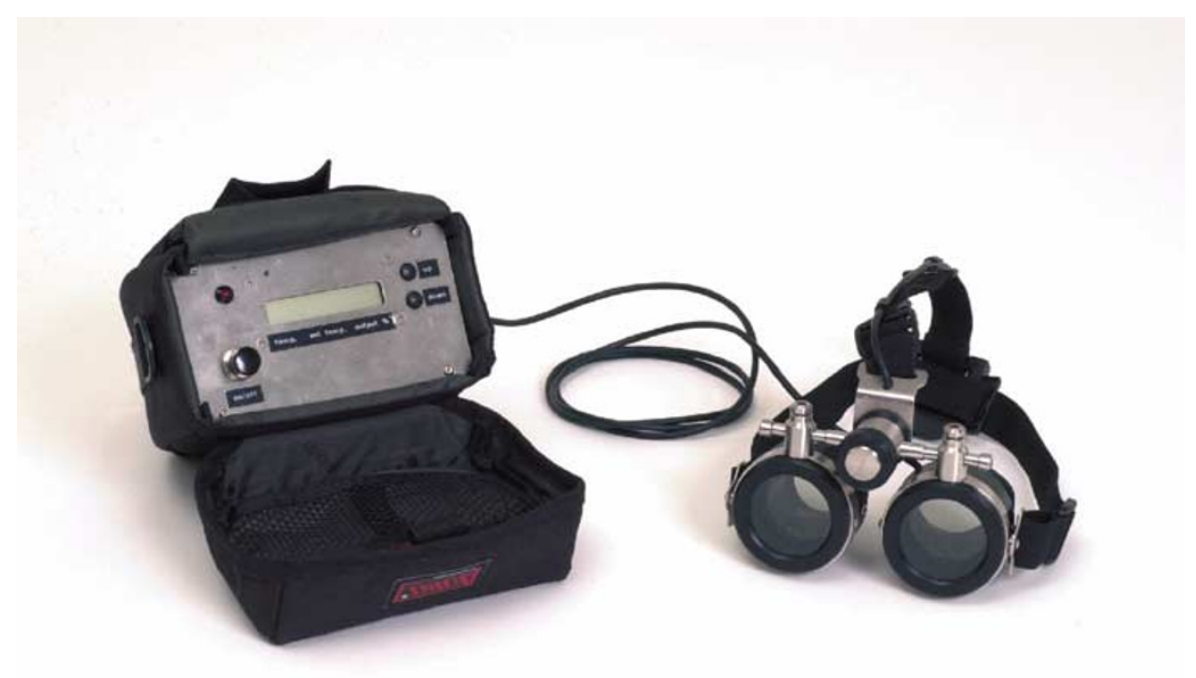

Figure 2 Prototype. 
Table 1 Changes in LLT and ocular comfort

\begin{tabular}{|c|c|c|c|c|c|c|c|c|}
\hline \multicolumn{2}{|l|}{ Patients } & \multicolumn{3}{|c|}{ Right eyes } & \multicolumn{3}{|c|}{ Left eyes } & \multirow{2}{*}{$\begin{array}{c}\text { Comfort } \\
\text { Subjective change }\end{array}$} \\
\hline Group & Number & LLT Pre-trial & LLT Post-trial & Change & LLT Pre-trial & LLT Post-trial & Change & \\
\hline & 1 & 4 & 4 & 0 & 4 & 5 & +1 & +1 \\
\hline Group I & 2 & 2 & 3 & +1 & 2 & 3 & +1 & 0 \\
\hline \multirow[t]{2}{*}{ Activated device } & 3 & 2 & 3 & +1 & 2 & 3 & +1 & +2 \\
\hline & 4 & 2 & 3 & +1 & 2 & 3 & +1 & +2 \\
\hline Mean LLT increase & 5 & 5 & 5 & 0 & 5 & 5 & 0 & 0 \\
\hline Left eyes 1.0 levels & 6 & 2 & 3 & +1 & 2 & 3 & +1 & +2 \\
\hline \multirow[t]{3}{*}{ Right eyes 0.9 levels } & 7 & 1 & 3 & +2 & 1 & 3 & +2 & +2 \\
\hline & 8 & 2 & 3 & +1 & 2 & 3 & +1 & +2 \\
\hline & 9 & 1 & 1 & 0 & 1 & 1 & 0 & 0 \\
\hline Group II & 10 & 2 & 2 & 0 & 2 & 2 & 0 & 0 \\
\hline \multirow[t]{2}{*}{ Nonactivated device } & 11 & 2 & 2 & 0 & 2 & 2 & 0 & 0 \\
\hline & 12 & 3 & 3 & 0 & 3 & 3 & 0 & 0 \\
\hline Mean LLT increase & 13 & 5 & 5 & 0 & 5 & 5 & 0 & 0 \\
\hline \multirow[t]{4}{*}{ Both eyes 0.1 levels } & 14 & 2 & 2 & 0 & 2 & 2 & 0 & 0 \\
\hline & 15 & 2 & 3 & +1 & 2 & 3 & +1 & +1 \\
\hline & 16 & 2 & 2 & 0 & 2 & 2 & 0 & 0 \\
\hline & 17 & 4 & 4 & 0 & 4 & 4 & 0 & 0 \\
\hline Group III & 18 & 2 & 2 & 0 & 2 & 2 & 0 & 0 \\
\hline \multirow[t]{2}{*}{ Controls } & 19 & 4 & 4 & 0 & 4 & 4 & 0 & 0 \\
\hline & 20 & 2 & 2 & 0 & 2 & 2 & 0 & 0 \\
\hline \multirow[t]{4}{*}{ No change in LLT } & 21 & 1 & 1 & 0 & 1 & 1 & 0 & 0 \\
\hline & 22 & 1 & 1 & 0 & 1 & 1 & 0 & 0 \\
\hline & 23 & 1 & 1 & 0 & 1 & 1 & 0 & 0 \\
\hline & 24 & 1 & 1 & 0 & 1 & 1 & 0 & 0 \\
\hline
\end{tabular}

LLT-Lipid layer thickness.

Grades of LLT: (1) open meshwork; (2) closed meshwork; (3) flow; (4) amorphous; (5) colour fringes of first order.

Change of the LLT is signified by a number corresponding to how many grades by which the LLT has changed. Thickening is denoted by a positive prefix; thinning by a negative prefix.

Subjective Comfort +3 markedly more comfortable; +2 moderately more comfortable; +1 mildly more comfortable; 0 no change; -1 mildly more uncomfortable; -2 moderately more uncomfortable; -3 markedly more uncomfortable.

\section{Group I}

Six subjects demonstrated bilaterally symmetrical increase in LLT (one level of increase in five subjects, two levels of increase in one individual). One subject exhibited a unilateral single-level increase in LLT. One subject (with the thickest pretreatment LLT) displayed no change. The mean increase for this group of subjects therefore measured 1.0 levels in left eyes and 0.9 levels in right eyes.

\section{Group II}

Seven subjects manifested no change in LLT. The remaining one subject had one level of increase in LLT in both eyes. The mean increase in LLT therefore measured 0.1 levels in both right and left eyes.

\section{Group III}

None of the participants demonstrated any change in LLT.
Subjects in Group I (treated) exhibited a significant increase in LLT compared with the other two groups (Kruskal-Wallis test, left eye $P<0.001$, right eye $P<0.003$ ). Follow-up analysis (Mann-Whitney test) showed no difference ( $P>0.3$ for both left and right eyes) between Groups II and III (inactive device and observation) but a significant difference between Groups I and II (active and inactive device) (left eyes $P<0.005$, right eyes $P<0.02$ ).

Of the eight subjects wearing the activated device (Group I), five reported a moderate increase in ocular comfort, one reported a minimal increase in comfort, while two experienced no difference. Six of eight subjects $(75 \%)$ thus experienced an improvement in ocular comfort. Of the eight subjects wearing the inactivated device (Group II), seven reported no change in ocular comfort. The one subject who reported minimal increase in comfort also exhibited an increase in LLT. No subject in the control group (Group III) experienced any subjective changes in comfort.

Overall seven of eight $(87.5 \%)$ subjects who developed thickening of the LLT reported improved comfort; 
conversely, no subject with an unchanged LLT reported any change in the level of comfort.

\section{Discussion}

Correlation between LLT and tear film stability has been demonstrated by observation of a four-fold increase in tear evaporation rate in the eye with an absent or nonconfluent lipid layer. ${ }^{1}$ Previous studies have also demonstrated correlation between LLT and two frequently used clinical tests, the tear film break-up time and Schirmer's test with anaesthesia.?

In our study, subjects in Group I (who wore the activated device) manifested statistically significant thickening of the lipid layer compared with the other two groups. All patients who exhibited thickening of the LLT (75\%) also experienced improvement in ocular comfort. Interestingly, the one subject wearing the nonactivated device who exhibited a binocular increase in LLT also reported improved comfort, the increase in LLT possibly having been related to simply enclosing the eyes and eyelids. ${ }^{8}$ Due to the intrinsically subjective nature of the comfort-level assessment, formal statistical analysis was not performed; it is however, noteworthy that $87.5 \%$ of subjects who exhibited increased LLT reported improved comfort. Conversely, no patient with an unchanged LLT reported any change in ocular comfort.

With regard to the wearing of the device, no complications were noted; neither was any discomfort reported.

The delivery of heat to the eyelids is an established therapy. ${ }^{9}$ Hot compresses are traditionally recommended for obstructive meibomian gland dysfunction and evaporative dry eye. Indeed, it has been shown that the melting point of meibomian gland secretions in subjects with meibomian gland dysfunction is $3^{\circ} \mathrm{C}$ higher than in normals, which may contribute to the solidification of such secretions in the context of meibomian gland dysfunction..$^{10}$ The use of hot compresses is, however, unpopular due to impracticality, underscoring the need to develop alternate modalities to deliver meibomian therapy with heat.

Noninflamed obstructive meibomian gland dysfunction has been shown to respond to thermotherapy with an infrared lid-heating device. ${ }^{11}$ However, this method, involving radiant heat, carries the risk of cataract formation. ${ }^{12,13}$ Our device delivers principally latent heat through a thermal siphon thereby avoiding the potential risks of radiant heat. Moreover, in contrast with hot compresses, our device allows normal lid movements that facilitate the release of meibomian secretions and resurfacing of the tear film. The longevity of such thickening of the lipid layer in response to a regular programme of meibomian therapy is well recognised. ${ }^{4}$

This study has shown that this device increases LLT and improves ocular comfort in normal subjects. Further studies are indicated to assess the efficacy of this device in patients with tear film and meibomian gland dysfunction.

Financial disclosure: Patent pending for device by ET Fuller and JR Fuller.

\section{References}

1 Craig JP, Tomlinson A. Importance of the lipid layer in human tear film stability and evaporation. Optom Vis Sci 1997; 74(1): 8-13.

2 Guillon M, Styles E, Guillon JP, Maissa C. Preocular tear film characteristics of non-wearers and soft contact lens wearers. Optom Vis Sci 1997; 74: 273-279.

3 Craig JP, Blades K, Patel S. Tear lipid layer structure and stability following expression of the meibomian glands. Ophthalmic Physiol Opt 1995; 15(6): 569-574.

4 Korb DR, Greiner JV. Increase in tear film lipid layer thickness following treatment of meibomian gland dysfunction. In: DA Sullivan (ed). Lacrimal Gland, Tear Film and Dry Eye Syndromes. Plenum Press: New York, 1994, pp 293-298.

5 Kanski JJ, Menon GJ. Clinical Ophthalmology, 5th edn. Butterworth Heinnemann: Oxford, 2003.

6 Efron N. Contact Lens Complications. Butterworth Heinemann: Oxford, 2002.

7 Isreb MA, Greiner JV, Korb DR, Glonek T, Mody SS, Finnemore VM et al. Correlation of lipid layer thickness measurements with fluorescein tear film break-up time and Schirmer's test. Eye 2003; 17(1): 79-83.

8 Korb DR, Craig J, Doughty M, Guillon JP, Smith G, Tomlinson A. The Tear Film Structure, Function And Clinical Examination. Butterworth Heineman: Oxford, 2002.

9 Duke-Elder S. Parsons' Diseases Of The Eye, 12th edn. Churchill: London, 1956.

10 Ong BL, Larke JR. Meibomian gland dysfunction: some clinical, biochemical and physical observations. Ophthal Physiol Opt 1990; 10(2): 144-148.

11 Goto E, Monden Y, Takano Y, Mori A, Shimmura S, Shimazaki J et al. Treatment of noninflamed obstructive meibomian gland dysfunction by an infrared warm compression device. Br J Ophthalmol 2002; 86(12): 1403-1407.

12 Okuno T. Thermal effect of infra-red radiation on the eye: a study based on a model. Ann Occup Hyg 1991; 35(1): 1-12.

13 Lydahl E, Philipson B. Infrared radiation and cataract. I. Epidemiologic investigation of iron- and steel-workers. Acta Ophthalmol 1984; 62(6): 961-975. 